\title{
Effect of a novel bladder preservation therapy, BOAI-CDDP-radiation (OMC-regimen)
}

\author{
HARUHITO AZUMA $^{1 *}$, TERUO INAMOTO $^{1 *}$, KIYOSHI TAKAHARA $^{1 *}$, HAYAHITO NOMI $^{1}$, HIROSHI UEHARA $^{1}$, \\ KAZUMASA KOMURA $^{1}$, KOICHIRO MINAMI ${ }^{1}$, JUNKO KOUNO ${ }^{1}$, YATSUGU KOTAKE ${ }^{3}$, HIROKAZU ABE ${ }^{4}$, \\ SHIZUKO TAKAGI $^{5}$, KAZUHIRO YAMAMOTO ${ }^{3}$, YOSHIHUMI NARUMI ${ }^{3}$ and SATOSHI KIYAMA $^{1}$
}

Departments of ${ }^{1}$ Urology and ${ }^{2}$ Radiology, Osaka Medical College, Takatsuki city, Osaka 569-8686; ${ }^{3}$ Department of Urology, Saiseikai Nakatsu Hospital, Osaka city, Osaka 530-0012; ${ }^{4}$ Department of Urology, Shizuoka Saiseikai Hospital, Shizuoka city, Shizuoka 422-8527; ${ }^{5}$ Department of Urology, Hirakata City Hospital, Hirakata city, Osaka 573-1013, Japan

Received March 1, 2013; Accepted April 12, 2013

DOI: 10.3892/ijo.2013.1923

\begin{abstract}
We have developed a novel form of bladder preservation therapy [OMC (Osaka Medical College)-regimen] involving balloon-occluded-arterial-infusion (BOAI) of an anticancer agent (cisplatin/gemcitabine), used concomitantly with hemodialysis, which delivers an extremely high concentration of anticancer agent to the site of a tumor without systemic adverse effects, along with concurrent radiation. We previously reported that the OMC-regimen elicited a complete response (CR) in $>90 \%$ of patients with organ confined tumors, while $\mathrm{LN}(+)$, T4 tumors and a non-UC histological type were statistically significant risk factors for treatment failure and patient survival. In this study, we investigated the effects of the OMC-regimen in patients with organ confined urothelial cancer tumors and the outcomes were compared to those with total cystectomy. Three hundred and one patients were assigned to receive either the OMC-regimen $(n=162)$ or total cystectomy $(n=139)$. Patients in the OMC-regimen group who failed to achieve CR underwent cystectomy, or secondary BOAI with an increased amount of CDDP or gemcitabine $(1600 \mathrm{mg})$. The OMC-regimen yielded $98.1 \%$ of clinical response; CR in $93.8 \%$ (152/162) of patients; PR in $4.3 \%$ (7/162). More than $96 \%$ of the CR patients (146/152) were
\end{abstract}

Correspondence to: Dr Haruhito Azuma, Department of Urology, Osaka Medical College, 2-7 Daigaku-cho, Takatsuki, Osaka 569-8686, Japan

E-mail: uro004@poh.osaka-med.ac.jp

${ }^{*}$ Contributed equally

Abbreviations: BOAI, balloon-occluded arterial infusion; CIS, carcinoma in situ; DSA, digital subtraction angiography; HD, hemodialysis; Qu, quartile; RTOG, radiation therapy oncology group; TURBT, transurethral resection of bladder tumor; UC, urothelial carcinoma

Key words: balloon-occluded-arterial-infusion, hemodialysis, invasive bladder cancer, trimodality therapy alive with no evidence of recurrence after a mean follow-up of 166 (range 23-960) weeks. No patients suffered grade III toxicity; all patients successfully completed this therapy. The patient survival was significantly better compared to the cystectomy group; the overall 5-, 10- and 15-year survival rates were $87.3,79.6$ and $59.7 \%$, respectively. Moreover, the 5-, 10- and 15-year bladder intact survival rates, the most important issue for bladder preservation therapy, were 85.7, 78.4 and $58.8 \%$, respectively. In conclusion, the OMC-regimen is a useful bladder-preservation strategy, not only in those for whom cystectomy is indicated, but also in patients whose condition is not amenable to curative treatment and for whom palliation would otherwise seem the only option.

\section{Introduction}

The standard method of treatment for patients with locally invasive bladder cancer is still a total cystectomy, which decrease the quality of life due to the urinary diversion and postoperative impotence. Reconstruction of the bladder using tissue from the intestinal tract has been applied in some cases and total cystectomy with nerve conservation in order to avoid postoperative impotence has been reported (1). However, these techniques require a long operation time and are invasive and are therefore not indicated for many patients because most cases of bladder cancer occur in individuals in their 60s-70s. In addition, the 5-year survival rates of patients with $\mathrm{pT} 2$, pT3a, pT3b and pT4 bladder cancer are 63-83, 50-69, 15-29 and $21-22 \%$, respectively (2). Approximately $50 \%$ of all patients with T2-T4 invasive bladder cancer die within 5 years despite of the decrease in QOL (3-10).

Trimodality therapy involving radical transurethral resection, chemotherapy and radiation therapy has long been attempted as an alternative approach for patients who require cystectomy. The Radiation Therapy Oncology Group (RTOG) has completed six prospective protocols entering 415 patients with T2-T4a muscle-invasive bladder cancer who were candidates for cystectomy. However, none of the protocols achieved a 5-year survival rate of $>60 \%$ (11-17). Moreover, the 5-year bladder intact survival rates were $<50 \%$ in most of studies (16-19). Improvement of the survival rate with bladder preser- 
vation may require a new method, which allows to deliver a higher dosage of anticancer agent specifically into the tumor, without causing systemic side effects.

We have therefore developed a novel bladder preservation therapy [referred to hereafter as the OMC (Osaka Medical College) regimen] involving balloon-occluded arterial infusion (BOAI) of an anticancer agent and concurrent hemodialysis (HD). This allows the anticancer agent to accumulate at a high concentration at the site of the tumor while ensuring that the systemic concentration remains low and this is then followed by radiation therapy.

We previously reported that $>90 \%$ of patients (70/77) with locally advanced urothelial bladder cancer who were treated in this way achieved CR, of whom 97\% (68/70) did not develop recurrent disease or metastasis within a mean follow-up period of 164 weeks [range, 11-805 weeks; 1st to 3rd quartile $(\mathrm{Qu})=66-195]$ after completion of therapy. In contrast to the high CR induction ratio in patients with locally invasive tumors, more than half of patients with lymph node involvement, stage T4 tumors and/or node involovement failed to achieve $\mathrm{CR}$ after the treatment.

To date, the number of patients who have received OMC-regimen is 221 , including 162 patients with organ confined (including T3b) urothelial cancer tumors. In this study we report the outcomes of the patients with organ confined (including T3b) urothelial cancer tumors treated with OMC-regimen in comparison with total cystectomy $(\mathrm{n}=139)$.

\section{Patients and methods}

Eligibility criteria. Eligible patients had histologically confirmed CIS, or stage T2, or T3 muscle-invasive bladder cancer without distant metastasis. Imaging studies, including chest computed tomography (CT) scan, abdominal/pelvic magnetic resonance imaging (MRI) and CT scan and bone scintigraphy were performed before the start of therapy. All patients who received the OMC-regimen had an absolute neutrophil count (ANC) of 1,500/ $\mu 1$, platelet count 100,000/ $\mu \mathrm{l}$, creatinine $3.0 \mathrm{mg} / \mathrm{dl}$, a bilirubin level 3 times the institutional upper limit of the normal range, an AST level 4 times the institutional upper limit of the normal range, an Eastern Cooperative Oncology Group (ECOG) performance status of $0-2$ and no prior radiotherapy or systemic therapy for bladder cancer. The study was reviewed and approved by the institutional review board of Osaka Medical College. Patients were informed of the investigational nature of the study and provided written informed consent before study enrollment.

Study design and treatment. Before study entry, patients underwent transurethral resection of the bladder tumor (TURBT) to establish the diagnosis. We primarily recommended total cystectomy when surgery was feasible. However, the OMC-regimen was offered as another treatment option whenever total cystectomy was not feasible because of advanced age, performance status or other reasons including patients' request. Patients were assigned to receive the OMC-regimen at 4-5 weeks after TURBT to allow adequate healing.

Assessability, toxicity and response criteria. Pretreatment evaluation included a complete history and physical examina-
Table I. Patient characteristics of both groups.

\begin{tabular}{|c|c|c|c|}
\hline Characteristic & $\begin{array}{l}\text { OMC- } \\
\text { regimen }\end{array}$ & $\begin{array}{c}\text { Total } \\
\text { cystectomy }\end{array}$ & P-value \\
\hline
\end{tabular}

\section{Age}

$\begin{array}{lrrr}\text { Median (range years) } & 70(39-91) & 65(44-89) & 0.0015 \\ \text { Sex } & & & \\ \text { Male (\%) } & 126(77.8 \%) & 116(83.5 \%) & \text { NS } \\ \text { Female (\%) } & 36(22.2 \%) & 23(16.5 \%) & \end{array}$

ECOG performance

status

$\begin{array}{rrrr}0 & 103(63.6 \%) & 90(64.7 \%) & \text { NS } \\ 1 & 46(28.4 \%) & 42(30.2 \%) & \text { NS } \\ 2 & 13(8.0 \%) & 7(5.1 \%) & \text { NS }\end{array}$

Clinical stage

T-stage

$\begin{array}{llll}\text { Cis } & 12(7.4 \%) & 11(7.9 \%) & \text { NS } \\ \text { T2 } & 91(56.2 \%) & 75(54.0 \%) & \text { NS } \\ \text { T3 } & 59(36.4 \%) & 53(38.1 \%) & \text { NS }\end{array}$

NS, not significant.

tion, performance status assessment, complete differential blood cell count, electrolytes, blood urea nitrogen, serum creatinine, liver function parameters and appropriate imaging studies to assess the extent of disease. During treatment, patients were seen weekly at our department, when their weight was recorded and toxicity was monitored using the National Cancer Institute's Common Terminology Criteria for adverse events v4.0 (CTCAE). At 10 weeks, patients underwent repeat transurethral resection of the site of the original tumor, ultrasound-guided whole-layer biopsy and urine cytology, as well as MRI and CT scan of the pelvis and were evaluated for their response to this therapy. CR was defined as complete disappearance of all measurable and evaluable disease. Duration of response was defined as the period from documentation of the response until evidence of disease recurrence. Survival was the period from study entry until patient death. Patients who achieved $\mathrm{CR}$ were observed using our follow-up protocol. However, any evidence of residual tumor in the bladder was deemed as treatment failure and such patients were primarily advised to undergo total cystectomy when possible, but otherwise to undergo secondary BOAI with a higher dosage of cisplatin or gemcitabine (1600 mg), as a salvage therapy. Patients who were found to have only a superficial amount of remaining tumor underwent intravesical injection of bacillus Calmette Guerin (BCG).

Follow-up. All patients were followed-up on the basis of monthly urine cytology, together with cystoscopy, biopsy and imaging studies, every three months for 2 years, including chest CT scan, abdominal/pelvic MRI and CT scan and bone scintigraphy and thereafter at 6-month intervals. 
Table II. Criteria for the administration of cisplatin.

In the initially enrolled 11 patients

$100 \mathrm{mg}$

$200 \mathrm{mg}$

Renal function $(\mathrm{sCr}>1.3)$ or age ( $\geq 75$ years)

$300 \mathrm{mg}$

Renal function ( $\mathrm{sCr}<1.3)$ with [age $(60-74$ years) and T-stage (T2 or T3)]

Renal function $(\mathrm{sCr}<1.3)$ with [age $(<60$ years $)$ or T-stage: $\mathrm{T} 4$ ]

In the latest 151 patients

$100 \mathrm{mg}$

All patients

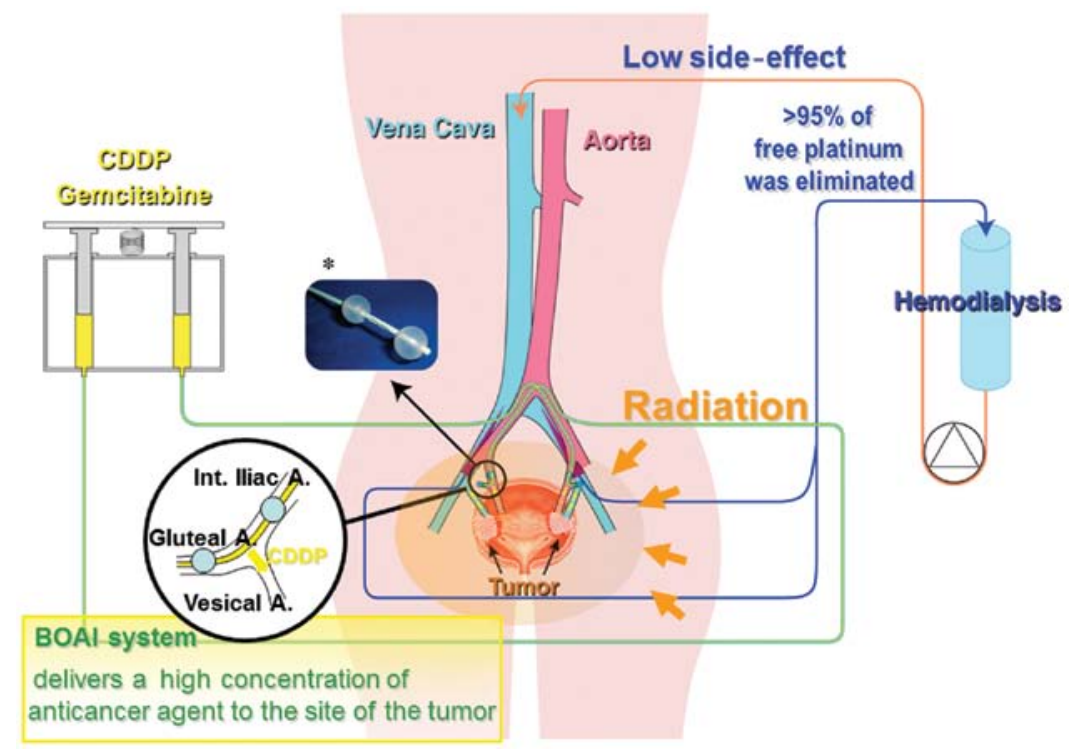

Figure 1. Schema of the OMC-regimen (HD-BOAI-CDDP/gemcitabine with radiation). The extracorporeal circuit allowed balloon-occluded intra-arterial infusion of CDDP/gemcitabine concurrent with HD. Through the femoral arterial approach, an intra-arterial catheter equipped with two occlusion balloons was introduced into the posterior trunk of the internal iliac artery on each side. Both the distal and proximal balloons were inflated and immobilized at a position allowing the 'vesical arteries' to be isolated between the balloons. The panel marked with an asterisk shows an image of the intra-arterial catheter (M6F-2870-TBSB4-ST, Clinical Supply), which is made of polyethylene, 6 French in size and equipped with two occlusion balloons separated by a distance of $40 \mathrm{~mm}$.

Statistics. Simple as well as multiple regression analyses were conducted to evaluate the significance of the following variables as risk factors of treatment failure: age, sex, patient performance status, tumor stage and tumor size and significance of complete resection of tumor and hydronephrosis due to tumors were also evaluated. The life table probabilities of overall survival and progression-free survival were determined using KaplanMeier analysis and log-rank test. The Cox proportional hazards regression analysis was conducted to assess the associations of each factor as described above. Differences at $\mathrm{P}<0.05$ were considered to be statistically significant.

\section{Results}

Patient characteristics. Between 1988 and 2013,162 (126 males and 36 females) were treated with the OMC-regimen and 139 (116 males and 23 females) underwent radical cystectomy. The characteristics of the patients in these two treatment groups are shown in Table I. For preoperative clinical staging, we used a simplified form of the 2002 TNM classification to stage bladder tumors (20). To make a valid comparison, preoperative clinical staging and not the pathologic stage after cystectomy was used to compare the two treatment groups, thus avoiding stage migration that may occur after pathologic staging (21).

\section{Treatment details}

i) OMC-regimen group. Patients assigned to the OMC-regimen group underwent transurethral resection of the bladder tumor (TURBT) at our institution to establish the diagnosis. They were then scheduled to receive the OMC-regimen 4-5 weeks after TURBT to allow adequate healing. We administered 100,200 , or $300 \mathrm{mg}$ of cisplatin as a single bolus, basically according to the criteria described in Table II.

For the intra-arterial infusion procedure, we used an intraarterial catheter equipped with two occlusion balloons (size: 6 Fr, M6F-28-70-TBSB4-ST, Clinical Supply, Tokyo, Japan). The catheter was introduced into the posterior trunk of the internal iliac artery through the femoral arterial approach and after the distal balloon had passed through the furcation of the anterior trunk of the internal iliac artery, both the distal and proximal balloons were inflated and immobilized, so that the anterior trunk of the internal iliac artery, which lies upstream of the target vessels (the vesical arteries) was isolated between the balloons. At this time, using digital subtraction angiography (DSA), it was confirmed that the injected agent did not enter the superior gluteal artery and that there was no back-flow into the internal iliac artery, while the tumor was markedly stained due to active flow of injected contrast medium into the urinary bladder. Fig. 1 illustrates the extracorporeal circuit used in the 
A

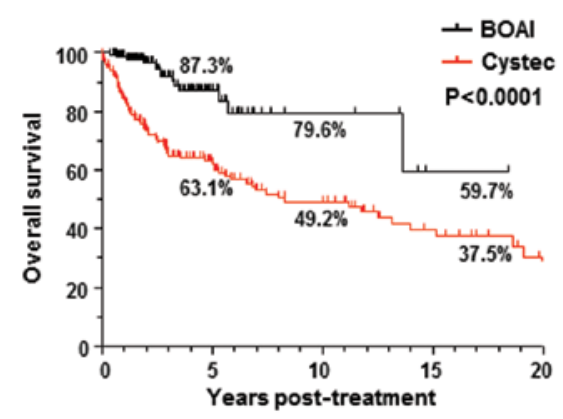

B

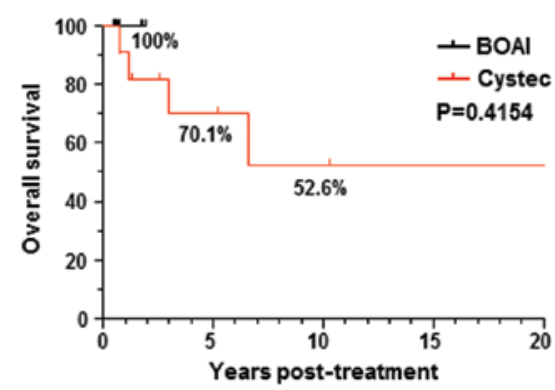

C

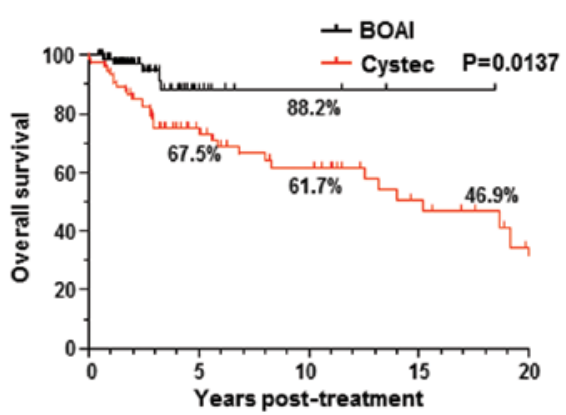

D

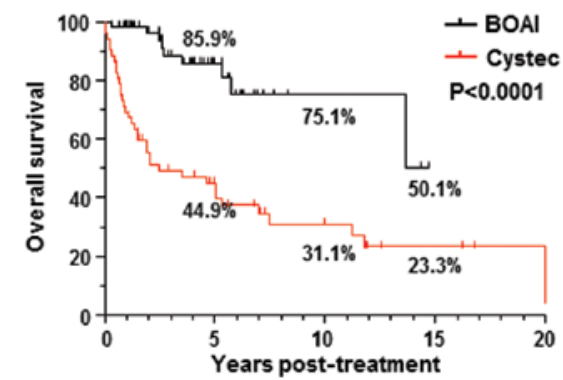

Figure 2. Comparison of overall survival between the OMC-regimen and cystectomy groups. Kaplan-Meier curves for overall survival in all patients (A), patients with tumor of CIS (B), T2 (C) and T3 (D) are shown respectively.

A

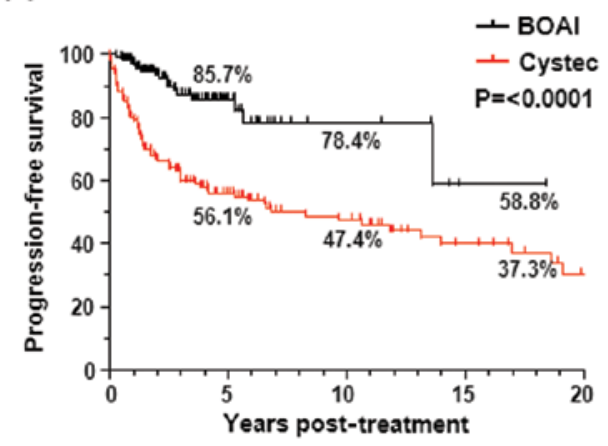

B

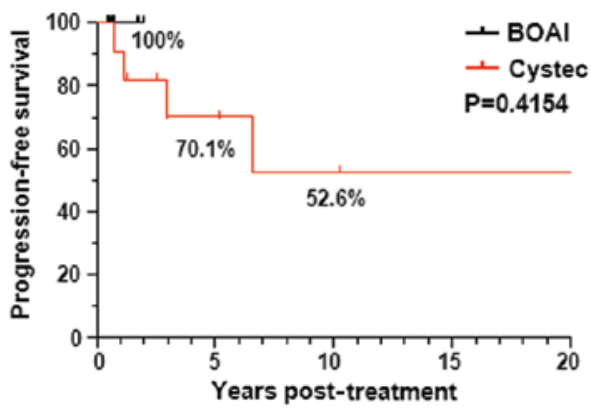

C

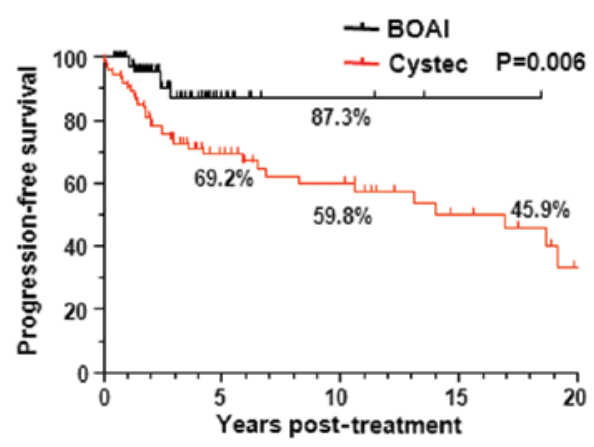

D

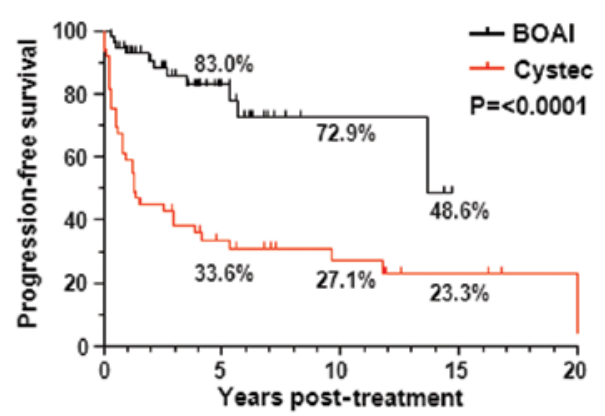

Figure 3. Comparison of progression-free survival between the OMC-regimen and cystectomy groups. Kaplan-Meier curves for overall survival in all patients (A), patients with tumor of CIS (B), T2 (C) and T3 (D) are shown respectively.

treatment. Various amounts of cisplatin $(100,200$ or $300 \mathrm{mg})$ were locally infused through the catheter over a 1-h period according to the criteria shown in Table II. Simultaneously, HD was performed via two double-lumen catheters (size:
$12 \mathrm{Fr}$, Argyle $^{\circledR}$, Tyco Healthcare, Tokyo, Japan) placed in the bilateral common iliac veins for $2 \mathrm{~h}$ after the start of arterial infusion. The catheters were connected to a hollow-fiber dialyzer (APS150, Asahi, Tokyo, Japan) with a membrane area 
of 1.0-1.5 $\mathrm{m}^{2}$ according to the weight of each patient. The blood flow rate was $180-250 \mathrm{ml} / \mathrm{min}$ and the hemodialysis-fluid flow rate was $500 \mathrm{ml} / \mathrm{min}$.

Radiation therapy was administered to the whole pelvis using a CT-planned three-dimensional conformal technique to a total of $60 \mathrm{~Gy}$ : $50 \mathrm{~Gy}$ ( 2 Gy/day x 25 days) followed by 10 Gy (2 Gy/day x 5 days) of local irradiation to the bladder. Patients were treated with the bladder empty. The planned target volume for the bladder included the gross target volume (bladder plus any extravesical tumor) with a 1-cm expansion. At 6 weeks, patients underwent repeat transurethral resection of the site of the original tumor, ultrasound-guided wholelayer biopsy and urine cytology, as well as MRI and CT scan of the pelvis and the response to this therapy was then evaluated.

ii) Radical cystectomy group. Among the 139 patients in the radical cystectomy group, 47 underwent ileal conduit formation, 58 underwent uretero-cutaneostomy, 19 and 7 patients underwent continent urinary diversion with ileal-neobladder formation (Hartmann's method) and Indiana pouch respectively and the remaining 6 underwent uretero-sigmoidostomy performed at the time of radical cystectomy. Standard pelvic lymphadenectomy was performed in 123 patients, 6 patients underwent iliac sampling and 10 patients were not studied in sufficient detail to allow assessment of the level of lymph node dissection. As not all of the histology reports mentioned the number of lymph nodes examined, it was not possible to precisely evaluate the extent of dissection. There were no significant differences in cause-specific or overall survival between the patients who underwent nodal dissection and the patients who did not. The urethrectomy was performed in 30 patients at the time of radical cystectomy because of the presence of extensive carcinoma in situ or multifocal bladder tumors.

Response to the OMC-regimen. Table III summarizes the treatment response, duration of response and patient characteristics, including sex, age, tumor stage, tumor size, involvement of hydronephrosis, performance status and success or failure of complete TURBT. Overall, 152 of the 162 patients (93.8\%) achieved a complete response as defined by the absence of persistent disease revealed by cystoscopy, biopsy and urine cytology after therapy. More than 96\% (146/152) of patients with CR were able to retain their urinary bladder with no evidence of recurrent disease or distant metastasis within a mean follow-up period of 168 weeks (range, 24-960 weeks; 1st to $3 \mathrm{rd} \mathrm{Qu}=54-234$ weeks) from the completion of therapy. The univariate as well as multivariate regression analyses revealed that a hydronephrosis is the only risk factor for treatment failure, while tumor stage, tumor size and failure of complete resection of tumor, those usually have been reported as risk factors for treatment failure of bladder preservation therapy, were not selected (Table IV).

\section{Comparison of patient survival between the two groups}

i) Overall survival $(O S)$. Overall survival ratio was significantly better in the OMC-regimen group than in the cystectomy group in all patients, the 5- and 15-year survival rates were 87.3 and 79.6 vs. 63.1 and $49.2 \%$, respectively; log-rank test, p $<0.0001$, Fig. 2A). Fig. 2B-D show OS ratio of patients in each T-stage of CIS, T2 and T3 respectively. The OS ratio in patients with T2 as well as T3 tumors was significantly better in the OMC-regimen group, although statistically significance has not seen in patients in with CIS (despite of $100 \%$ of PFS), because of short follow-up period (Fig. 3).

i) Progresion-free survival (PFS). The progression-free survival ratio was also significantly better in the OMC-regimen group in total patients, as well as in each patient with $\mathrm{T} 2$ and T3 tumors. In all patients, $>85$ and $78 \%$ of patients survive with their functioning bladder at 5 and 10 years, respectively; this is the most important issue for the bladder preservation therapy.

Predictors of patient survival selected using univariate and multivariate Cox regression analyses in OMC-regimen group. We investigated the significance of each factor, including sex, age, tumor stage, tumor size, involvement of hydronephrosis, performance status and success or failure of complete TURBT as a predictor of overall survival and progression-free survival using the Cox regression model. As shown in Table V, univariate Cox regression analysis as well as multivariate Cox regression analysis selected only the hydronephrosis as a significant factor affecting both progression-free and overall survival, while tumor stage, tumor size and failure of complete resection of tumor were not selected.

Toxicity. The observed toxicities are summarized in Table VI. It is noteworthy that none of the patients had grade II or more severe blood/bone marrow and/or gastrointestinal toxicities. Ten patients $[6.17 \%, 95 \%$ confidence interval (CI), 3.0-11.1\%] experienced grade I blood/bone marrow toxicity, 21 (13.0\%, 95\% CI, 8.21-19.1\%) had gastrointestinal toxicity and 6 (3.7\%, 95\% CI, 1.37-7.89\%) had neuropathy. The duration of blood/bone marrow toxicity, including granulocytopenia and anemia, was relatively short: median duration was 5 days for granulocytopenia (range 3-9 days) and 3 days for anemia (range 2-10 days). No patients received granulocyte colony-stimulating factor or transfusion of red blood cells. Gastrointestinal toxicity included anorexia in 16 patients, constipation in 7 , diarrhea in 9 , nausea in 14 and vomiting in 2, but all symptoms disappeared within 6 -days after intraarterial infusion. One patient experienced grade II neuropathy in the peroneal nerve area, but disappeared by the 12 months after the treatment. There were no other adverse reactions such as renal failure, genitourinary toxicity, radiation cystitis or life-threatening complications.

\section{Discussion}

We have previously reported that the OMC-regimen allowed $>89 \%(69 / 77)$ of patients with locally invasive tumors to achieve CR [ $>70 \%(70 / 96)$ of all patients including those with T4 and $\mathrm{N}(+)$ disease]. Most (68/69) of the CR patients were still alive with no evidence of recurrence after a mean follow-up of 161 (range 12-805) weeks. The 5- and 15-year overall survival rates were 91.5 and $81.3 \%$ (vs. 59.8 and $40.1 \%$ for cystectomy, $\mathrm{P}<0.0001$ ), respectively. In contrast to the high CR induction ratio in patients with locally invasive tumors, more than half 


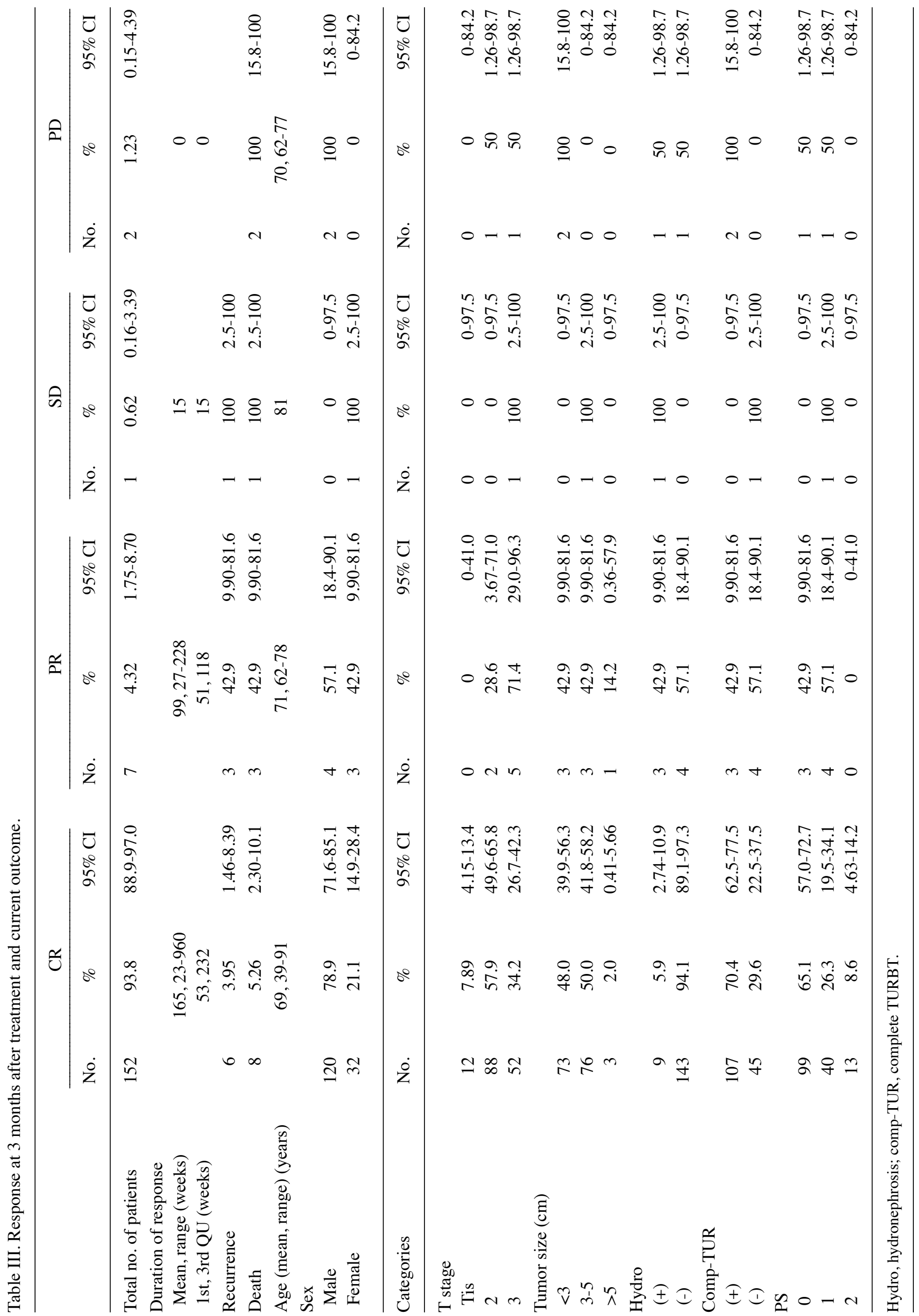


Table IV. Risk factors for treatment failure in the OMC-regimen group.

\begin{tabular}{|c|c|c|c|c|c|}
\hline & \multirow[b]{2}{*}{ Category } & \multicolumn{2}{|c|}{ Univariate } & \multicolumn{2}{|c|}{ Multivariate } \\
\hline & & Odds ratio & P-value & Odds ratio & P-value \\
\hline Hydronephrosis & (+) vs. (-) & 15.87 & 0.0001 & 71.43 & 0.0013 \\
\hline T-stage (Tis) & Cis vs. $\mathrm{T} 2$ & 1969 & 0.9753 & NV & NV \\
\hline T-stage (T3) & T3 vs. T2 & 3.953 & 0.0537 & NV & NV \\
\hline Tumor size & $>3 \mathrm{~cm}$ vs. $<3 \mathrm{~cm}$ & 1.082 & 0.9037 & 1.823 & 0.1322 \\
\hline Tumor size & $>5 \mathrm{~cm}$ vs. $<5 \mathrm{~cm}$ & 5.325 & 0.1562 & 7.576 & 0.5583 \\
\hline Tumor number & Cont. variable & 1.175 & 0.6717 & 2.785 & 0.2967 \\
\hline Complete TUR & Yes vs. no & 2.378 & 0.1873 & 1.359 & 0.5118 \\
\hline Performance status & 2 vs. $0-1$ & 10598 & 0.9726 & 207067 & 0.9964 \\
\hline Sex & Male vs. female & 2.500 & 0.1749 & 2.809 & 0.2401 \\
\hline Age & Cont. variable & 1.034 & 0.3286 & 1.062 & 0.1985 \\
\hline
\end{tabular}

Cont. variable, continuous variable; NV, no value.

Table V. Predictors of overall survival (A) and progression-free survival (B) in the OMC-regimen group evaluated by univariate and multivariate Cox regression analyses.

A, Overall survival

\begin{tabular}{|c|c|c|c|c|c|}
\hline & \multirow[b]{2}{*}{ Category } & \multicolumn{2}{|c|}{ Univariate } & \multicolumn{2}{|c|}{ Multivariate } \\
\hline & & Odds ratio & P-value & Odds ratio & P-value \\
\hline Hydronephrosis & (+) vs. (-) & 14.29 & $<0.0001$ & 21.74 & 0.0004 \\
\hline T-stage & T3 vs. T2 & 1.570 & 0.4282 & NV & NV \\
\hline Tumor size & $>3 \mathrm{~cm}$ vs. $<3 \mathrm{~cm}$ & 1.382 & 0.5991 & 2.976 & 0.2989 \\
\hline Tumor size & $>5 \mathrm{~cm}$ vs. $<5 \mathrm{~cm}$ & 5.208 & 0.0194 & 4.785 & 0.1159 \\
\hline Tumor number & Cont. variable & 1.020 & 0.9534 & 1.135 & 0.7229 \\
\hline Complete TUR & Yes vs. no & 1.668 & 0.3954 & 1.287 & 0.7846 \\
\hline Performance status & 2 vs. $0-1$ & 1.026 & 0.9805 & 1.299 & 0.8361 \\
\hline Sex & Male vs. female & 2.129 & 0.1977 & 1.546 & 0.5300 \\
\hline Age & Cont. variable & 1.012 & 0.7086 & 1.004 & 0.9224 \\
\hline
\end{tabular}

B, Progression-free survival

\begin{tabular}{|c|c|c|c|c|c|}
\hline & \multirow[b]{2}{*}{ Category } & \multicolumn{2}{|c|}{ Univariate } & \multicolumn{2}{|c|}{ Multivariate } \\
\hline & & Odds ratio & P-value & Odds ratio & P-value \\
\hline Hydronephrosis & (+) vs. (-) & 9.524 & $<0.0001$ & 13.51 & 0.0002 \\
\hline T-stage & T3 vs. T2 & 1.838 & 0.2100 & NV & NV \\
\hline Tumor size & $>3 \mathrm{~cm}$ vs. $<3 \mathrm{~cm}$ & 1.350 & 0.5566 & 1.709 & 0.4855 \\
\hline Tumor size & $>5 \mathrm{~cm} \mathrm{vs} .<5 \mathrm{~cm}$ & 4.255 & 0.0551 & 2.545 & 0.3166 \\
\hline Tumor number & Cont. variable & 1.051 & 0.8611 & 1.092 & 0.7685 \\
\hline Complete TUR & Yes vs. no & 1.246 & 0.6604 & 1.166 & 0.8307 \\
\hline Performance status & 2 vs. $0-1$ & 1.387 & 0.6650 & 2.232 & 0.3730 \\
\hline Sex & Male vs. female & 1.712 & 0.2899 & 1.362 & 0.6008 \\
\hline Age & Cont. variable & 1.003 & 0.9159 & 1.007 & 0.8217 \\
\hline
\end{tabular}

Cont. variable, continuous variable; NV, no value. 
Table VI. Toxicity.

\begin{tabular}{|c|c|c|c|c|c|c|}
\hline \multirow[b]{2}{*}{ Toxicity } & \multicolumn{3}{|c|}{ Grade } & \multicolumn{3}{|c|}{ Duration } \\
\hline & $\begin{array}{l}\text { Grade } 1 \\
\text { No. }(\%)\end{array}$ & $\begin{array}{l}\text { Grade } 2 \\
\text { No. }(\%)\end{array}$ & $\begin{array}{c}\text { Grade 3-4 } \\
\text { No. }(\%)\end{array}$ & $\begin{array}{c}<3 \text { days } \\
\text { No. }\end{array}$ & $\begin{array}{c}\text { 3-7 days } \\
\text { No. }\end{array}$ & $\begin{array}{c}>7 \text { days } \\
\text { No. }\end{array}$ \\
\hline \multicolumn{7}{|l|}{ Blood/bone marrow } \\
\hline Total & $10(6.2)$ & $3(1.9)$ & 0 & & & \\
\hline Granulocytopenia & $5(3.1)$ & 0 & 0 & 0 & $5(3.1)$ & 0 \\
\hline Anemia & $7(4.3)$ & $3(1.9)$ & 0 & $3(1.9)$ & $4(2.5)$ & 0 \\
\hline \multicolumn{7}{|l|}{ Gastrointestinal } \\
\hline Total & $21(13.0)$ & 0 & 0 & & & \\
\hline Anorexia & $16(9.9)$ & 0 & $11(6.8)$ & $5(3.1)$ & 0 & \\
\hline Constipation & 7 (4.3) & 0 & 0 & $6(3.7)$ & $1(0.6)$ & 0 \\
\hline Diarrhea & $9 \quad(5.6)$ & 0 & 0 & $4(2.5)$ & $5(3.1)$ & 0 \\
\hline Nausea & 14 (8.6) & 0 & 0 & $9(5.6)$ & $5(3.1)$ & 0 \\
\hline Vomiting & $2(1.2)$ & 0 & 0 & 0 & $2(1.2)$ & 0 \\
\hline Neuropathy & $6(3.7)$ & $1(0.6)$ & 0 & 0 & 0 & $7(4.3)$ \\
\hline
\end{tabular}

of the patients with lymph node involvement, stage T4 tumors and/or node involovement failed to achieve CR after the treatment. Stage T4, lymph node involvement and histological type (non-UC) were independent statistically significant risk factors for treatment failure as well as patient survival.

To date, the number of patients who have received OMC-regimen is 221, including 162 patients with organ confined (including T3b) urothelial cancer tumors and other patients with histologically non-UC tumors and/or stage T4 tumors and/or node involvement. In this study we focused on the patients with locally invasive urothelial cancer and report the outcome. The treatment allowed $>93 \%$ of patients to achieve CR after the treatment. More than $96 \%$ of the patients with CR have been able to retain their bladder without recurrence or distant metastasis, with a mean survival of 168 weeks. This supports our previous data, which indicated that patients with locally invasive urothelial bladder cancer are the best candidates for this therapy.

The most pertinent issue related to this therapy is why it achieves better outcomes than cystectomy. Using a rat BOAI-CDDP model, we are now investigating the mechanisms responsible for the better outcomes achieved by the OMC-regimen in comparison with total cystectomy. We have found that BOAI allows delivery of an extremely high concentration of anticancer agent to the bladder and surrounding pelvic region, as well as to the pelvic and para-aortic lymphatic tissues (data not shown). Enhanced radiosensitivity of the cancer cells due to the BOAI-induced high concentration of cisplatin in the bladder, as well as in the lymphatic tissues, may also have contributed significantly to the good outcomes achieved. In addition, the severe hypoxia in the target region resulting from BOAI may play a role in the marked antitumor effect, as several basic studies have demonstrated that hypoxia greatly enhances the effectiveness of cisplatin (22-24). Future studies may help to clarify the key roles of BOAI, thus contributing to further improvements of this therapy.
The other advantage of the OMC-regimen is a significant reduction of systemic side effects. Cisplatin exerts its antitumor activity via the non-protein-bound form, whose concentration falls steeply after administration: its half-life is normally $<60 \mathrm{~min}$ and its concentration falls to below the detection limit $4 \mathrm{~h}$ after administration $(25,26)$. The most important point of the OMC-regimen is the removal of non-protein-bound $\mathrm{Pt}$ immediately after administration of cisplatin by performing HD via the bilateral common iliac veins, thus accomplishing efficient drainage of cisplatin immediately after passage through the tumor. HD is specifically efficient for cisplatin elimination, since the molecular weight of protein-unbound cisplatin is $\sim 300$, similar to that of creatinine. Moreover, the anatomic structure and blood supply of the bladder may largely account for the efficient drainage of cisplatin achieved with this approach. As the urinary bladder is situated at the base of the pelvis, the relatively close circuit formed by the internal iliac artery, bladder and common iliac veins may contribute to efficient drainage of the anticancer agent, thus increasing the elimination efficiency without influencing the systemic circulation. Indeed, we found that $>95 \%$ of free Pt was efficiently eliminated by HD during BOAI of cisplatin, thus providing optimal conditions for effective local accumulation of $\mathrm{Pt}$ in the tumor, with minimal systemic toxicity, allowing even a 91-year-old patient to complete the regimen.

Thus, the OMC-regimen, which delivers an extremely high concentration of anticancer agent to the site of a tumor without causing systemic adverse effects, can be regarded as a curative therapy for elderly patients, not only those for whom total cystectomy is indicated, but also those of whom total cystectomy is not feasible because of age, performance status or other reasons and who are considered physically incapable of tolerating the chemotherapeutic regimens that are usually applied clinically. It is noteworthy that this therapy will improve the feasibility of radical cure without the need for cystectomy in patients for whom such surgery would other- 
wise be necessary and also facilitate potential cure in patients whose condition would normally rule out this likelihood and for whom, otherwise, merely palliative treatment would seem the only option.

\section{References}

1. Schlegel PN and Walsh PC: Neuroanatomical approach to radical cystoprostatectomy with preservation of sexual function. J Urol 138: 1402-1406, 1987.

2. Pagano F, Bassi P, Galetti TP, et al: Results of contemporary radical cystectomy for invasive bladder cancer: a clinicopathological study with an emphasis on the inadequacy of the tumor, nodes and metastases classification. J Urol 145: 45-50, 1991.

3. Stenzl A, Cowan NC, De Santis M, et al: Guidelines on bladder cancer: muscle-invasive and metastatic. Eur Assoc Urol 3: 1-59, 2008.

4. Hong S, Kwak C, Jeon H, Lee E and Lee S: Do vascular, lymphatic, and perineural invasion have prognostic implications for bladder cancer after radical cystectomy? Urology 65: 697-702, 2005.

5. Leissner J, Koeppen C and Wolf H: Prognostic significance of vascular and perineural invasion in urothelial bladder cancer treated with radical cystectomy. J Urol 169: 955-960, 2003.

6. Patton S, Hall M and Ozen H: Bladder cancer. Curr Opin Oncol 14: 265-272, 2002.

7. Shelley MD, Barber J and Wilt T: Surgery versus radiotherapy for invasive bladder cancer. Cochrane Database Syst Rev: CD002079, 2002.

8. Stein JP, Lieskovsky G, Cote R, et al: Radical cystectomy in the treatment of invasive bladder cancer: Long-term results in 1,054 patients. J Clin Oncol 19: 666-675, 2001.

9. Dalbagni G, Genega E, Hashibe M, et al: Cystectomy for bladder cancer: a contemporary series. J Urol 165: 1111-1116, 2001.

10. Frazier H, Robertson J, Dodge R and Paulson D: The value of pathologic factors in predicting cancer-specific survival among patients treated with radical cystectomy for transitional cell carcinoma of the bladder and prostate. Cancer 71: 3993-4001, 1993.

11. Tran E, Souhami L, Tanguay S and Rajan R: Bladder conservation treatment in the elderly population: results and prognostic factors of muscle-invasive bladder cancer. Am J Clin Oncol 32 333-337, 2009.

12. Weizer AZ, Joshi D, Daignault S, et al: Performance status is a predictor of overall survival of elderly patients with muscle invasive bladder cancer. J Urol 177: 1287-1293, 2007.

13. Hoshi S, Shintaku I, Suzuki K, et al: Bladder preservation by internal iliac arterial infusion chemotherapy and irradiation in T3 bladder carcinoma patients over the age of 70 years. Tohoku J Exp Med 192: 249-258, 2000.

14. Kaufman DS, Winter KA, Shipley WU, et al: The initial results in muscle-invading bladder cancer of RTOG 95-06: phase I/II trial of transurethral surgery plus radiation therapy with concurrent cisplatin and 5-fluorouracil followed by selective bladder preservation or cystectomy depending on the initial response. Oncologist 5: 471-476, 2000.
15. Rodel C, Grabenbauer GG, Kuhn R, et al: Combined-modality treatment and selective organ preservation in invasive bladder cancer: long-term results. J Clin Oncol 20: 3061-3071, 2002.

16. Shipley WU, Winter KA, Kaufman DS, et al: Phase III trial of neoadjuvant chemotherapy in patients with invasive bladder cancer treated with selective bladder preservation by combined radiation therapy and chemotherapy: initial results of Radiation Therapy Oncology Group 89-03. J Clin Oncol 16: 3576-3583, 1998.

17. Tester W, Caplan R, Heaney J, et al: Neoadjuvant combined modality program with selective organ preservation for invasive bladder cancer: results of Radiation Therapy Oncology Group phase II trial 8802. J Clin Oncol 14: 119-126, 1996.

18. Hagan MP, Winter KA, Kaufman DS, et al: RTOG 97-06: initial report of a phase I-II trial of selective bladder conservation using TURBT, twice-daily accelerated irradiation sensitized with cisplatin, and adjuvant MCV combination chemotherapy. Int J Radiat Oncol Biol Phys 57: 665-672, 2003.

19. Kaufman DS, Winter KA, Shipley WU, et al: Phase I-II RTOG study (99-06) of patients with muscle-invasive bladder cancer undergoing transurethral surgery, paclitaxel, cisplatin, and twicedaily radiotherapy followed by selective bladder preservation or radical cystectomy and adjuvant chemotherapy. Urology 73: 833-837, 2009

20. Greene FL, Page DL and Fleming ID: AJCC Cancer Staging Manual. 6th edition. Springer Verlag, New York, NY, 2002.

21. Ficcara V, Dalpiaz O, Alrabi N, Novara G, Galfano A and Artibani W: Correlation between clinical and pathological staging in a series of radical cystectomies for bladder carcinoma. BJU Int 95: 786-790, 2005.

22. Douple EB and Richmond RC: A review of platinum complex biochemistry suggests a rationale for combined platinum-radiotherapy. Int J Radiat Oncol Biol Phys 8: 1335-1339, 1979.

23. Douple EB and Richmond RC: Radiosensitization of hypoxic tumor cells by cis- and trans-dichlorodiammineplatinum (II). Int J Radiat Oncol Biol Phys 8: 1369-1372, 1979.

24. Abbott DW, Freeman ML and Holt JT: Double-strand break repair deficiency and radiation sensitivity in BRCA2 mutant cancer cells. J Natl Cancer Inst 90: 978-985, 1998.

25. Belt RJ, Himmelstein KJ, Patton TF, Bannister SJ, Sternson LA and Repta AJ: Pharmacokinetics of nonprotein-bound platinum species following administration of cis-dichlorodiammineplatinum(II). Cancer Treat Rep 63: $1515-1521,1979$

26. Himmelstein KJ, Patton TF, Belt RJ, Taylor S, Repta AJ and Sternson LA: Clinical kinetics on intact cisplatin and some related species. Clin Pharmacol Ther 29: 658-664, 1981. 